\title{
REVIEW
}

Open Access

\section{Rituximab for non-infectious Uveitis and Scleritis}

Caleb C. Ng ${ }^{1,2^{*}}$, Aileen Sy ${ }^{1,3}$ and Emmett T. Cunningham Jr $r^{1,2,4,5}$

\begin{abstract}
Purpose: To provide a comprehensive review of rituximab use for the treatment of non-infectious uveitis and scleritis.

Methods: Review of literature through December 2020.

Results: Individual data was available for 229 patients with refractory non-infectious uveitis $(n=108)$ or scleritis $(n=121)$ who received treatment with rituximab (RTX). Rituximab was generally utilized as third-line or later treatment (uveitis: 67/90, 74.4\%; scleritis: 90/96, 93.8\%) at a mean of 33.5 months following the diagnosis of uveitis (range $=0$ to 168.0 months; median $=24.0$ months) and 39.4 months after diagnosis of scleritis (range $=1.0$ to 168.0 months; median $=21.0$ months). Patients with non-infectious uveitis and scleritis either received prior treatment with corticosteroids only (uveitis: 18/90, 20\%; scleritis: 4/94, 4.3\%), or with one (uveitis: 19/90, 21.1\%; scleritis: 30/94, 31.9\%), two (uveitis: 11/90, 12.2\%; scleritis 27/94, 28.7\%), or three or more (uveitis: 37/90, 41.1\%; scleritis: 31/94, 33.0\%) corticosteroid-sparing immunosuppressive agents with or without corticosteroids before initiation of RTX treatment. The rheumatologic protocol (two infusions of $1 \mathrm{gram}$ of RTX separated by 14 days) was utilized most frequently (uveitis: 45/87, 51.7\%; scleritis: 87/114, 76.3\%), followed by the Foster protocol (eight weekly infusions of 375 mg/m² RTX; uveitis: 18/87, 20.7\%; scleritis: 10/114, 8.8\%), and the oncologic protocol (four weekly infusions of $375 \mathrm{mg} / \mathrm{m}^{2}$ RTX; uveitis: 5/87, 5.7\%; scleritis: 6/114, 5.3\%). Various other off-label regimens were used infrequently (uveitis: 19/87, 21.8\%; scleritis 11/114, 9.6\%). Rituximab treatments resulted in a positive therapeutic response for the majority of patients with non-infectious uveitis $(81 / 97,83.5 \%)$. Commonly treated uveitic diagnoses included nonparaneoplastic autoimmune retinopathy (30/107, 28.0\%), juvenile idiopathic arthritis (21/107, 19.6\%), Vogt-Koyanagi-Harada disease (12/107, 11.2\%), and Behçet disease (11/107, 10.3\%). Cases of non-infectious scleritis were most commonly attributed to granulomatosis with polyangiitis $(75 / 121,62.0 \%)$ and rheumatoid arthritis $(15 / 121,12.4 \%)$, and showed an even greater rate of positive therapeutic response (112/120, 93.3\%) following RTX treatment. No side effects were reported in 76.3\% (74/ 97) of uveitis and $85.5 \%$ (71/83) scleritis cases. Of those cases associated with RTX-induced adverse events, the most common were infusion reactions of various severity $(11 / 35,31.4 \%)$.
\end{abstract}

Conclusions: Overall, RTX appeared to be both effective and well-tolerated as second or third-line therapy for patients with non-infectious uveitis and scleritis.

Keywords: Autoimmune retinopathy, Intraocular inflammation, Rituxan

\footnotetext{
* Correspondence: Calebctng@gmail.com

'Department of Ophthalmology, California Pacific Medical Center, San

Francisco, CA, USA

${ }^{2}$ West Coast Retina Medical Group, 1445 Bush Street, San Francisco, CA

94109, USA

Full list of author information is available at the end of the article
}

\section{Springer Open}

(c) The Author(s). 2021 Open Access This article is licensed under a Creative Commons Attribution 4.0 International License, which permits use, sharing, adaptation, distribution and reproduction in any medium or format, as long as you give appropriate credit to the original author(s) and the source, provide a link to the Creative Commons licence, and indicate if changes were made. The images or other third party material in this article are included in the article's Creative Commons licence, unless indicated otherwise in a credit line to the material. If material is not included in the article's Creative Commons licence and your intended use is not permitted by statutory regulation or exceeds the permitted use, you will need to obtain permission directly from the copyright holder. To view a copy of this licence, visit http://creativecommons.org/licenses/by/4.0/. 


\section{Introduction}

While corticosteroids are the first-line therapy for noninfectious uveitis and scleritis, their prolonged use is limited by common side effects [1-3]. Patients requiring long-term immunosuppression for severe disease have been traditionally treated with non-corticosteroid immunosuppressive agents such as methotrexate or azathioprine, leukocyte inhibitors such as cyclosporine or tacrolimus, or with alkylating agents such as cyclophosphamide or chlorambucil [1, 2, 4, 5]. For ocular inflammation refractory to these more traditional therapies, practitioners have increasingly turned to use of biologic agents such as intravenous immunoglobulin, interferons, and tumor necrosis factor (TNF) antagonists [5, 6]. Rituximab (RTX; Rituxan ${ }^{\odot}$, Genentech, South San Francisco, CA, USA), a fully humanized anti-CD20 antibody, has gained increasing use and acceptance for the treatment noninfectious ocular inflammation refractory to corticosteroids and traditional immunotherapies. Rituximab causes a depletion of B cells for up to 6 months and is approved by the Food and Drug Administration (FDA) for treatment of nonHodgkin's lymphoma, chronic lymphocytic leukemia, rheumatoid arthritis (RA), and granulomatosis with polyangiitis (GPA, formerly Wegener's Granulomatosis) and microscopic polyangiitis [6-8]. Use of RTX has been described in over 100 cases each of refractory non-infectious uveitis and scleritis, as summarized below.

\section{Methods}

The authors conducted a literature search using the National Library of Medicine's PubMed database for all English language articles published through December 2020 with the following search terms: "rituximab AND eye", "rituximab AND uveitis," and "rituximab AND scleritis." Use of RTX for orbital inflammation was summarized in a separate companion review [9]. Relevant references within these articles were also reviewed. Included here were all cases of non-infectious scleritis and uveitis for which individual case data was available. Articles describing large series of patients in which individual case data was not provided were not included in the current analysis, but were read for content and referenced when appropriate. Individual information on patient age, sex, anatomical localization and cause of disease, treatment prior to initiation of RTX, time from diagnosis to initiation of RTX, visual acuity before RTX treatment began, visual acuity at last visit, RTX treatment regimen, total number of RTX cycles and interval between cycles, therapeutic response, treatments following initiation of RTX therapy, duration of follow-up and whether disease recurrence occurred, and adverse events attributed to RTX were collected when available. Therapeutic response varied from study to study. In this review, a patient was considered to have had a positive therapeutic response to RTX if they achieved disease quiescence, showed a two-step or more improvement in inflammation as graded by Standardization of Uveitis Nomenclature group [10], showed relative or absolute sparing of corticosteroids or non-corticosteroid medications, or if the authors subjectively documented improvement. Line of therapy was tallied according to the following criteria: first-line - RTX initiated before or at same time as corticosteroids; second-line - RTX initiated after corticosteroids - either alone or with a second more traditional immunosuppressive agent; third-line or greater - RTX initiated after a non-corticosteroid immunosuppressive agent, such as nonbiologic or biologic disease modifying antirheumatic drug, alkylating agents, or intravenous immunoglobulins, with or without corticosteroids. Treatment regimens were classified into either the rheumatologic protocol (two doses of $1000 \mathrm{mg}$ separated by 14 days) [11], the oncologic protocol (four doses of $375 \mathrm{mg} / \mathrm{m}^{2}$ weekly) [12], the Foster protocol (eight doses of $375 \mathrm{mg} / \mathrm{m}^{2}$ weekly) [13], or other category for the less commonly utilized dosing protocols. Univariate comparisons were made with two-tailed Ttest and nomimal, uncorrected $p$-values were reported.

\section{Results}

\section{Uveitis (Tables 1, 2)}

There have been a total of 31 reports describing 108 patients who received treatment with RTX for noninfectious uveitis $[14,15,16,17,18,19,20,21,22,23$, $24,25,26,27,28,29,30,31-45]$. There was a male to female ratio of 0.26 to 1 , and patients possessed a mean age of $39.8 \pm 19.4$ years (range $=8.0$ to 86.0 years; median $=40.0$ years). The anatomical location of uveitis was most commonly posterior uveitis (57/108, 52.8\%), followed by anterior uveitis (30/108, 27.8\%), panuveitis $(13 / 108,12.0 \%)$, unspecified uveitis $(4 / 108,3.7 \%)$, anterior and intermediate uveitis $(2 / 108,1.9 \%)$, intermediate uveitis $(1 / 108,0.9 \%)$, and anterior and posterior uveitis (1/108, $0.9 \%)$. Underlying systemic conditions included non-paraneoplastic autoimmune retinopathy (npAIR; 30/107, 28.0\%), juvenile idiopathic arthritis (JIA; 21/107, $19.6 \%)$, Vogt-Koyanagi-Harada (VKH) disease (12/107, $11.2 \%)$, Behçet disease $(11 / 107,10.3 \%)$, cancer-associated retinopathy (CAR; 10/107, 9.3\%), sarcoidosis (4/107, $3.7 \%)$, GPA (3/107, 2.8\%), systemic lupus erythematosus (SLE; 2/107, 1.9\%), indeterminate etiology (9/107, 8.4\%), and one case each $(1 / 107,0.9 \%)$ of melanoma-associated retinopathy (MAR), type 2 essential cryoglobulinemia, birdshot chorioretinopathy (BSCR), Human Leukocyte Antigen (HLA)-B27-associated uveitis, and multiple sclerosis. Among the 57 cases $(47.1 \%)$ that reported preRTX vision, 38.6\% (22/57) had vision worse than 20/200, whereas $31.6 \%(18 / 57)$ had vision better than or equal to 20/40, and $29.8 \%$ (17/57) had vision between $20 / 40$ and $20 / 200$. 
Table 1 Rituximab use in refractory non-infectious uveitis and scleritis - summary of comprehensive literature review

\begin{tabular}{lll}
\hline & Non-Infectious Uveitis & Non-Infectious Scleritis \\
\hline Number of Studies & 31 & 36 \\
Total Patients & 108 & 121 \\
Age & Mean $39.8 \pm 19.4$ & Mean $48.5 \pm 16.6$ \\
(years) & Median 40.0 & Median 52.0 \\
& Range $8.0-86.0$ & Range $16.0-81.0$ \\
Gender & $0.26: 1(\mathrm{M}: \mathrm{F})$ & $0.56: 1(\mathrm{M}: \mathrm{F})$ \\
Ocular Condition & Posterior uveitis $(57 / 108,52.8 \%) ;$ & Anterior scleritis $(71 / 121,58.7 \%) ;$ \\
Treated with Rituximab & Anterior uveitis (30/108, 27.8\%); & Episcleritis (7/121,5.7\%); \\
(Ratio, \%) & Panuveitis (13/108, 12.0\%); & Posterior scleritis $(7 / 121,5.7 \%) ;$ \\
& Unspecified uveitis $(4 / 108,3.7 \%) ;$ & Anterior and posterior scleriti $(3 / 121,2.5 \%) ;$ \\
& Anterior and intermediate uveitis $(2 / 108,1.9 \%) ;$ & Location unspecified $(33 / 121,27.3 \%)$
\end{tabular}

Underlying Systemic

Condition

(Ratio, \%)

Treatment Prior to Rituximab

(Ratio, \%)

Previously Utilized Immunosuppressants (Ratio, \%)

Line of Therapy

(Ratio, \%)

Treatment Regimen and Number of Cycles
Anterior and posterior uveitis (1/108, 0.9\%);

npAIR (30/107, 28.0\%) [14-22];

Juvenile idiopathic arthritis (21/107, 19.6\%) [23-26];

Vogt-Koyanagi-Harada disease (12/107, 11.2\%) [27-30]; Behçet disease (11/107, 10.3\%) [31, 32];

Cancer-associated retinopathy (10/107, 9.3\%) [14,

33-36];

Sarcoidosis (4/107, 3.7\%) [37];

Granulomatosis with polyangiitis $(3 / 107,2.8 \%)$ [38-

40];

Systemic lupus erythematosus $(2 / 107,1.9 \%)[40]$

Birdshot chorioretinopathy (1/107, 0.9\%) [41]

HLA-B27 (1/107, 0.9\%) [40];

Multiple sclerosis (1/107, 0.9\%) [42];

Melanoma-associated retinopathy $(1 / 107,0.9 \%)$ [14];

Type 2 essential cryoglobulinemia (1/107, 0.9\%) [43]; Indeterminate $(9 / 107,8.4 \%)[24,40,44,45]$

Corticosteroid only $(18 / 90,20.0 \%)$

1 Steroid sparing agent (19/90, 21.1\%);

2 Steroid sparing agents $(11 / 90,12.2 \%)$

$\geq 3$ Steroid sparing agents (37/90, 41.1\%);

None $(5 / 90,5.6 \%)$

Corticosteroids (69/85, 81.2\%);

methotrexate (39/85, 45.9\%);

cyclosporine $(30 / 85,35.3 \%)$;

adalimumab $(24 / 85,28.2 \%)$;

infliximab (24/85, 28.2\%);

mycophenolate mofetil $(24 / 85,28.2 \%)$;

etanercept $(15 / 85,17.6 \%)$;

azathioprine $(11 / 85,12.9 \%)$;

IVIG (8/85, 9.4\%);

chlorambucil $(4 / 85,4.7 \%)$;

leflunomide (4/85, 4.7\%);

cyclophosphamide $(3 / 85,3.5 \%)$;

hydroxychloroquine $(3 / 85,3.5 \%)$

tacrolimus $(2 / 85,2.4 \%)$;

abatacept $(2 / 85,2.4 \%)$

anakinra $(1 / 85,1.2 \%)$

sulfasalazine (1/85, 1.2\%);

interferon-alpha $(1 / 85,1.2 \%)$;

bortezomib (1/85, 1.2\%)

First line $(5 / 90,5.6 \%)$;

Second line $(18 / 90,20.0 \%)$;

Third or greater $(67 / 90,74.4 \%)$

Rheumatologic protocol (45/87, 51.7\%);

Other (19/87, 21.8\%);
GPA (75/121, 62.0\%) $[11,24,38,39,46-66]$;

Rheumatoid arthritis $(15 / 121,12.4 \%)[48,55,60,67-$ 70];

ANCA-associated vasculitis, NOS (5/121, 4.1\%) [37];

GPA and Rheumatoid arthritis (3/121, 2.5\%) [56];

GPA and lgG4-related disease (1/121, 0.8\%) [71];

Microscopic polyangiitis (1/121, 0.8\%) [60];

Sjogren's syndrome $(1 / 121,0.8 \%)[12] ;$

HLA-B27 (1/121, 0.8\%) [40];

Behcet's disease (1/121, 0.8\%) [24];

Mixed connective tissue disease

and Scleroderma (1/121, 0.8\%) [55];

IgG4-related disease (1/121, 0.8\%) [72];

Cogan syndrome $(1 / 121,0.8 \%)[48]$;

Indeterminate (15/121, 12.4\%) [48, 55, 60, 70, 73, 74]

Corticosteroid only (4/94, 4.3\%)

1 Steroid sparing agent $(30 / 94,31.9 \%)$

2 Steroid sparing agents $(27 / 94,28.7 \%)$

$\geq 3$ Steroid sparing agents (31/94, 33.0\%);

None: $(2 / 94,2.1 \%)$

Corticosteroids (62/90, 68.9\%);

cyclophosphamide (55/90, 61.1\%);

methotrexate (42/90, 46.7\%);

mycophenolate mofetil (24/90, 26.7\%);

azathioprine (24/90, 26.7\%);

infliximab $(12 / 90,13.3 \%)$;

NSAID $(11 / 90,12.2 \%)$;

etanercept $(10 / 90,11.1 \%)$;

adalimumab (9/90, 10.0\%);

cyclosporine (8/90, 8.9\%);

leflunomide (6/90, 6.7\%);

hydroxychloroquine (3/90, 3.3\%);

IVIG (2/90, 2.2\%);

sulfasalazine (2/90, 2.2\%);

doxycycline (2/90, 2.2\%);

Unspecified anti-TNF agent (2/90, 2.2\%);

anakinra $(1 / 90,1.1 \%)$;

minocycline (1/90, 1.1\%);

interferon alpha $(1 / 90,1.1 \%)$;

golimumab (1/90, 1.1\%);

Abatacept (1/90, 1.1\%);

Chlorambucil (1/90, 1.1\%);

Tocilizumab (1/90, 1.1\%);

Cytarabine $(1 / 90,1.1 \%)$

First line: $(2 / 96,2.1 \%)$;

Second line: $(4 / 96,4.2 \%)$;

Third line: (90/96, 93.8\%)

Rheumatologic protocol (87/114, 76.3\%); Other $(11 / 114,9.6 \%)$; 
Table 1 Rituximab use in refractory non-infectious uveitis and scleritis - summary of comprehensive literature review (Continued)

\begin{tabular}{|c|c|c|}
\hline & Non-Infectious Uveitis & Non-Infectious Scleritis \\
\hline (Ratio, \%) & $\begin{array}{l}\text { Foster protocol }(18 / 87,20.7 \%) \\
\text { Oncologic protocol }(5 / 87,5.7 \%) \\
1 \text { treatment cycle: }(27 / 88,30.7 \%) \\
2 \text { treatment cycles: }(26 / 88,29.5 \%) \\
>2 \text { treatment cycles: }(35 / 88,39.8 \%)\end{array}$ & $\begin{array}{l}\text { Foster protocol }(10 / 114,8.8 \%) ; \\
\text { Oncologic protocol }(6 / 114,5.3 \%) \\
1 \text { treatment cycle: }(34 / 95,35.8 \%) ; \\
2 \text { treatment cycles: }(18 / 95,18.9 \%) ; \\
\text { >2 treatment cycles: }(43 / 95,45.3 \%)\end{array}$ \\
\hline $\begin{array}{l}\text { Types of Responses } \\
\text { (Ratio, \%) }\end{array}$ & $\begin{array}{l}\text { Responsive }(81 / 97,83.5 \%) \\
\text { - Disease Remission }(51 / 81,63.0 \%) \\
\text { - Author report: }(30 / 81,37.0 \%) \\
\text { Nonresponsive }(16 / 97,16.5 \%)\end{array}$ & $\begin{array}{l}\text { Responsive }(112 / 120,93.3 \%) \\
\text { - Disease Remission: }(99 / 112,88.4 \%) \\
\text { - Author report: }(13 / 112,11.6 \%) \\
\text { Non-responsive }(8 / 120,6.7 \%)\end{array}$ \\
\hline $\begin{array}{l}\text { Incidence of Recurrence } \\
\text { (Ratio, \%) }\end{array}$ & $24 / 81,29.6 \%$ & $33 / 107,30.8 \%$ \\
\hline $\begin{array}{l}\text { Adverse Events } \\
\text { (Ratio, \%) }\end{array}$ & 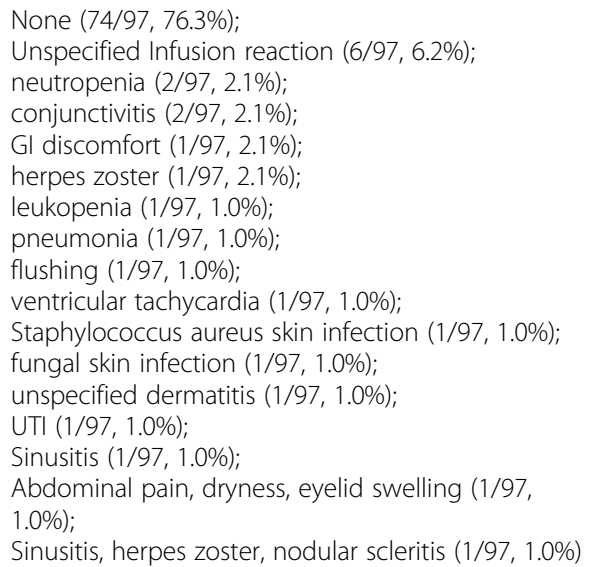 & $\begin{array}{l}\text { None }(71 / 83,85.5 \%) ; \\
\text { Itching (infusion reaction) }(2 / 83,2.4 \%) ; \\
\text { Disease exacerbation }(2 / 83,2.4 \%) ; \\
\text { Pneumonia and septic shock }(1 / 83,1.2 \%) ; \\
\text { Herpetic mouth ulcers }(1 / 83,1.2 \%) ; \\
\text { Acute retinal necrosis }(1 / 83,1.2 \%) ; \\
\text { Herpes zoster }(1 / 83,1.2 \%) ; \\
\text { Hypotension (Infusion reaction) }(1 / 83,1.2 \%) ; \\
\text { Ocular hypertension (1/83, 1.2\%); } \\
\text { Dry eyes, weight loss, fatigue, and joint pain (1/83, } \\
1.2 \%) ; \\
\text { Bronchitis, bacterial pneumonia, } \\
\text { leukopenia, and anemia }(1 / 83,1.2 \%)\end{array}$ \\
\hline
\end{tabular}

RTX rituximab, $M$ male, $F$ female, npAIR non-paraneoplastic autoimmune retinopathy, GPA granulomatosis with polyangiitis, NOS not otherwise specified, HLA human leukocyte antigen, ANCA Anti-Neutrophilic Cytoplasmic Autoantibody, NOS not otherwise specified, Rheumatologic Two doses of 1000 mg separated by 14 days, Oncologic four doses of $375 \mathrm{mg} / \mathrm{m} 2$ weekly, Foster eight doses of $375 \mathrm{mg} / \mathrm{m} 2$ weekly, Other all other RTX dosing regimens, NSAID nonsteroidal antiinflammatory drug, IVIG intravenous immunoglobulin, TNF tumor necrosis factor, First line RTX initiated before or as same time as corticosteroids, Second line RTX initiated after corticosteroids, Third line RTX initiated after corticosteroids and another agent, such as nonbiologic or biologic disease modifying antirheumatic drug, anti-cancer medications, or intravenous immunoglobulins

In the 90 cases with documented treatment history, a total of $74.4 \%$ (67/90) of patients received RTX as thirdline or later therapy, followed by $20.0 \%(18 / 90)$ as second-line, and 5.6\% (5/90) as first-line. Previous therapies included none $(5 / 90,5.6 \%)$, corticosteroids alone $(18 / 90,20 \%)$, or one $(19 / 90,21.1 \%)$, two $(11 / 90,12.2 \%)$, or three or more $(37 / 90,41.1 \%)$ corticosteroid-sparing immunosuppressive agents with or without corticosteroids. Immunosuppressive agents tried prior to RTX included corticosteroids $(69 / 85,81.2 \%)$, methotrexate (39/ $85,45.9 \%)$, cyclosporine $(30 / 85,35.3 \%)$, adalimumab (24/85, 28.2\%), infliximab (24/85, 28.2\%), mycophenolate mofetil $(24 / 85,28.2 \%)$, etanercept $(15 / 85,17.6 \%)$, azathioprine $(11 / 85,12.9 \%)$, intravenous immunoglobulins $(8 /$ $85,9.4 \%)$, chlorambucil $(4 / 85,4.7 \%)$, leflunomide $(4 / 85$, $4.7 \%)$, hydroxychloroquine $(3 / 85,3.5 \%)$, cyclophosphamide $(3 / 85,3.5 \%)$, tacrolimus $(2 / 85,2.4 \%)$, abatacept $(2 /$ $85,2.4 \%)$, anakinra $(1 / 85,1.2 \%)$, sulfasalazine $(1 / 85$, $1.2 \%)$, interferon-alpha $(1 / 85,1.2 \%)$, and bortezomib (1/ $85,1.2 \%)$. The mean time from diagnosis to RTX use was $33.5 \pm 34.7$ months (range $=0$ to 168.0 months; median $=24.0$ months).
Various RTX treatment regimens were utilized to treat patients with non-infectious uveitis, with 51.7\% (45/87) receiving the rheumatologic protocol, $21.8 \%(19 / 87)$ receiving a variety of uncommon off-label protocols, $20.7 \%$ (18/87) receiving the Foster protocol, and 5.7\% (5/87) receiving the oncologic protocol. A total of $30.7 \%(27 / 88)$ patients received just 1 cycle of RTX, leading to disease remission for $81.5 \%$ (22/27), no observed response for $18.5 \%(5 / 27)$, and eventual disease recurrence in $54.5 \%$ of responders $(12 / 22)$ at a mean of 8.0 months (range $=$ 6.0 to 13.0 months; median $=7.0$ months) despite continued treatment with other forms of systemic immunomodulatory therapies. In total, $69.3 \%(61 / 88)$ of treated patients received between two to 12 cycles of RTX at varying intervals: 4 weeks $(20 / 58,34.5 \%)[16,17,37,38$, 40], 8 weeks $(3 / 58,5.2 \%)[17,37], 3$ to 6 months $(1 / 58$, $1.7 \%)$ [38], 6 months $(25 / 58,43.1 \%)[17,20,22,23,29$, 30] 8 months (1/58 1.7\%) [25], 9 months $(1 / 58,1.7 \%)$ [25], 6 to 10 months $(1 / 58,1.7 \%)$ [24], or $\geq 12$ months $(4 / 58,6.9 \%)[15,17,25]$. Two studies employed increasing retreatment durations $(2 / 58,3.4 \%)[27,28]$. Three studies utilized the Foster protocol, followed by four 


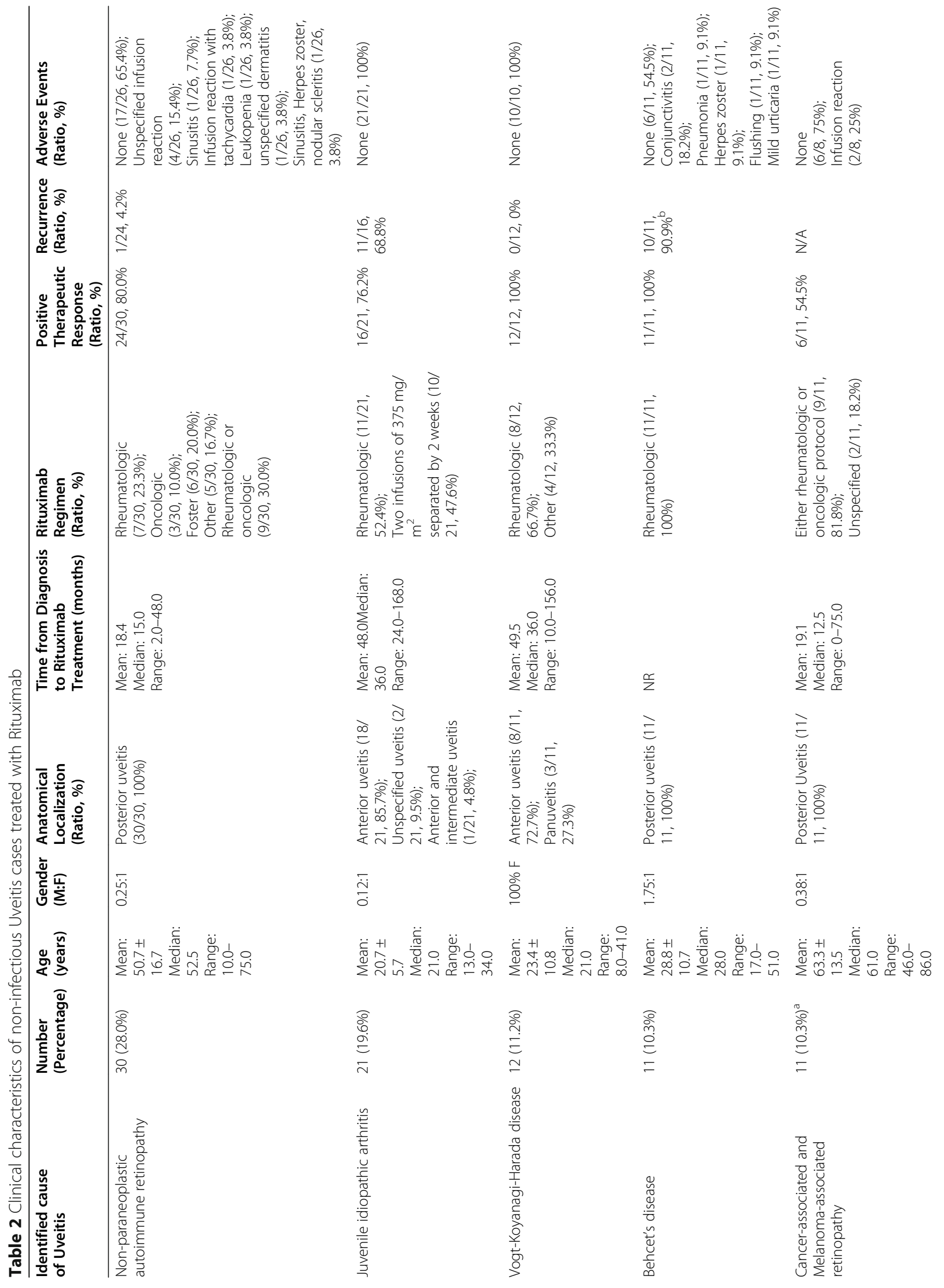




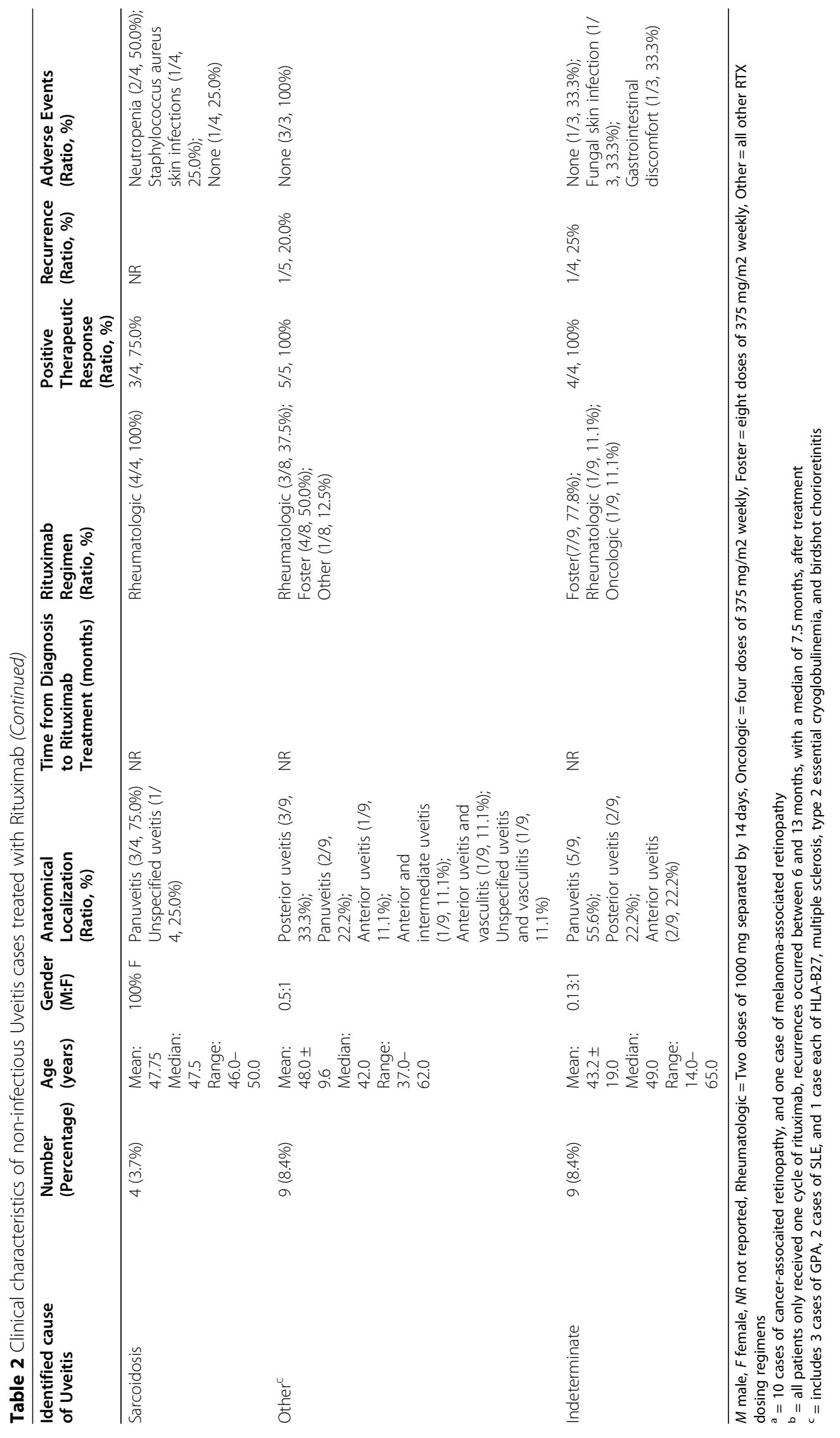


monthly infusions at the same dose, and then further monthly treatments as needed based on clinical examination, but did not provide individual data on total number of treatments and indication for each retreatment $[16,38,40]$. For the patients with available longitudinal data $(47 / 61,77.0 \%), 23.4 \%$ (11/47) received two or more cycles of RTX for disease recurrence [23-25, 38]. Otherwise, the rationale of designated treatment intervals and indications for retreatment were unclear in the other reviewed studies.

In reports with available individual data $(n=97)$, 83.5\% (81/97) of patients treated with RTX experienced a positive therapeutic response, with $63.0 \%(51 / 81)$ having disease remission and 37.0\% (30/81) showing disease improvement or stability based on author report. In contrast, $16.5 \%$ (16/97) of patients were described as having treatment failure with RTX. One or more disease recurrences eventually developed in $29.6 \%$ (24/81) of the patients responsive to RTX, with mean interval to first disease recurrence of 8.5 months (range $=3$ to 17 months; median $=8.0$ months). Information on individualized treatment regimens following uncomplicated RTX therapy was available for $78.9 \%$ (45/57) of patients who experienced disease remission without recurrence, with $44.4 \%(20 / 45)$ receiving ongoing treatment with one or more non-RTX, corticosteroid-sparing systemic immunosuppressive agents at mean follow up of 17.6 months (range $=1.0$ to 36.0 months; median $=18.0$ months), $29.3 \%(12 / 45)$ sustaining RTX treatments at mean follow up of 17.1 months (range $=7.0$ to 60.0 months; median $=17.1$ months), $17.8 \%(8 / 45)$ achieving drug-free remission at mean follow up of 32.6 months (range $=4$ to 81 months; median $=22.0$ months), and $11.1 \%(5 / 45)$ maintaining disease remission with only topical corticosteroid therapy at a mean follow up 14.2 months $\quad$ (range $=11$ to 26 months; median $=11.0$ months). Among the patients with reported visual acuities following RTX therapy, 39.2\% (20/51) had vision between $20 / 40$ and $20 / 200,35.3 \%(18 / 51)$ better than or equal to $20 / 40$, and $25.5 \%(13 / 51)$ were worse than or equal to $20 / 200$.

A total of $76.3 \%$ (74/97) of patients who received RTX treatments for non-infectious uveitis reported no adverse events. However, six cases $(6 / 97,6.2 \%)$ of unspecified infusion reactions $[14,20]$, two cases each $(2 / 97,2.1 \%)$ of neutropenia [37] and conjunctivitis [31], and one case each $(1 / 97,1.0 \%)$ of gastrointestinal discomfort [45], herpes zoster [31], sinusitis [14], leukopenia [14], pneumonia [31], flushing during infusion [31], ventricular tachycardia during infusion [14], Staphylococcus aureus skin infection [37], fungal skin infection [44], unspecified dermatitis [16], and urinary tract infection [40] were reported following RTX treatments. Two individuals reported multiple adverse events attributed to RTX; one
(1/97, 1.0\%) developed abdominal pain, dryness, and eyelid swelling, and the other $(1 / 97,1.0 \%)$ sinusitis, herpes zoster, and nodular scleritis $[17,40]$.

\section{Non-paraneoplastic autoimmune retinopathy (npAIR)}

Patients with npAIR accounted for $28.0 \%$ (30/107) of patients with non-infectious uveitis who received treatment with RTX. There was a 0.25 to 1 male to female ratio, and mean age at time of treatment was 50.7 years (range $=10.0$ to 75.0 years; median $=52.5$ years). The mean interval from diagnosis of npAIR to initiation of RTX treatment was 18.4 months (range $=2.0$ to 48.0 months; median $=$ 15.0 months). One study with nine patients $(9 / 30,30.0 \%)$ utilized either the rheumatologic or oncologic protocols, but did not specify [14]. Otherwise, 23.3\% (7/30) of cases were treated according to the rheumatologic protocol, $20 \%(6 / 30)$ the Foster protocol, $16.7 \%$ (5/30) with various uncommon off-label treatment regimens, and 10.0\% (3/ 30 ) the oncologic protocol. A total of $80.0 \%(24 / 30)$ of RTX-treated patients were described as having disease remission or regression following RTX treatment due to improvement or stability in vision, visual fields, and/or electroretinography (ERG). One treatment responder (1/ 24, 4.2\%) developed later disease relapse. Mild infusion reactions $(4 / 26,15.4 \%)[14,20]$ were the most common reported adverse event, but sinusitis [14], tachycardia following infusion [14], leukopenia [14], unspecified dermatitis [16], and the combination of sinusitis, herpes zoster, and nodular scleritis [17] were reported in one patient each $(1 / 26,3.8 \%)$.

\section{Cancer-associated retinopathy (CAR) and melanoma- associated retinopathy (MAR)}

In total, $10.3 \%$ of patients (11/107; 10 CAR, 1 MAR) with non-infectious uveitis were treated with RTX for paraneoplastic retinopathy. These patients had a male to female ratio of 0.38 to 1 and mean age of 63.3 years (range $=46.0$ to 86.0 years; median 61.0 years) when RTX treatment began. The mean interval from diagnosis of CAR or MAR to initiation of RTX treatment was 19.1 months (range $=0$ to 75.0 months; median $=12.5$ months). A total of $81.8 \%$ (9/11) of patients received treatment with either the rheumatologic or oncologic protocol, while the dose and frequency was unspecified in $18.2 \%(2 / 11)$. Following RTX treatment, $54.5 \%(6 / 11)$ of cases were assessed to have a positive therapeutic response based on improvement in vision, visual fields, and/or ERG. Overall, $75.0 \%(6 / 8)$ reported no adverse events following RTX treatments, but unspecified infusion reactions were reported in two patients $(2 / 8,25.0 \%)[14]$.

\section{Juvenile idiopathic arthritis}

A total of $19.6 \%$ (21/107) of patients who received RTX for non-infectious uveitis possessed a systemic diagnosis 
of JIA. These patients possessed a male to female ratio of 0.12 to 1 and mean age of 20.7 years (range $=13.0$ to 34.0 years, median $=21.0$ years) at time of treatment initiation. The mean interval from diagnosis of uveitis to treatment with RTX was 48.0 months (range $=24.0$ to 168.0 months; median $=36.0$ months). Overall, $85.7 \%$ $(18 / 21)$ possessed anterior uveitis, 9.5\% (2/21) unspecified uveitis, and $4.8 \%(1 / 21)$ anterior and intermediate uveitis. In total, $52.4 \%(11 / 21)$ of patients received treatment with the rheumatologic protocol, and $47.6 \%$ (10/ 21) with a modified rheumatologic protocol using 375 $\mathrm{mg} / \mathrm{m}^{2}$ RTX dosing instead of $1 \mathrm{~g}$. Following treatment with RTX, 76.2\% (16/21) of recipients experienced a positive therapeutic response, but $68.8 \%$ (11/16) of responders eventually developed disease recurrence. No adverse events were reported in this subgroup.

\section{Vogt-Koyanagi-Harada (VKH) disease}

Patients with VKH disease accounted for 11.2\% (12/107) of patients with non-infectious uveitis who were treated RTX. All patients were female and possessed a mean age of 23.4 years (range $=8.0$ to 41.0 years; median $=21.0$ years) at time of RTX therapy. The mean interval from initial diagnoses of uveitis to RTX treatment was 49.5 months (range $=10.0$ to 156.0 months; median $=36.0$ months $)$. In total, $72.7 \%(8 /$ 11) of patients were diagnosed with anterior uveitis and $27.3 \%$ (3/11) with panuveitis. A total of $66.7 \%$ (8/12) were treated according to the rheumatologic protocol, and the remaining $33.3 \%$ (4/12) with a variety of uncommon offlabel regimens. All subjects received two or more doses without any reported adverse events and were described to achieve disease remission without relapse at mean follow up of 19.8 months (range $=9.0$ to 36.0 months; median = 17.0 months).

\section{Behçet disease}

In total, 10.3\% (11/107) of patients who received RTX for non-infectious uveitis possessed various forms of posterior uveitis caused by Behçet disease. Gender distribution was $63.6 \%$ (7/11) male and 36.4\% (4/11) female, and mean age at time of treatment was 28.8 years (range $=17.0$ to 51.0 years; median $=28.0$ years). All patients were treated with just 1 cycle of the rheumatologic protocol $(11 / 11,100 \%)$ and were reported to have a positive therapeutic response $(11 / 11,100 \%)$ with disease remission, but there was a $90.9 \%(10 / 11)$ rate of subsequent disease relapse occurring at mean interval of 8.2 months $\quad$ (range $=6.0$ to 13.0 months; median $=7.5$ months). Overall, $54.5 \%(6 / 11)$ of treated patients reported no adverse events, but two patients each $(2 / 11$, 18.2\%) developed conjunctivitis and mild infusion reactions, and one patient each $(1 / 11,9.1 \%)$ developed unspecified pneumonia and herpes zoster attributed to RTX [31].

\section{Uveitis of indeterminate etiology}

A total of $8.4 \%(9 / 107)$ of patients with non-infectious uveitis who received RTX treatment possessed no discernable systemic etiology for their ocular inflammation. Gender distribution was $88.9 \%$ (8/9) female and $11.1 \%$ (1/9) male, and the mean age at time of RTX therapy was 43.2 years $($ range $=14.0$ to 65.0 years; median $=49.0$ years). In total, $55.6 \%$ (5/9) of patients possessed panuveitis and $22.2 \%(2 / 9)$ each possessed posterior uveitis and anterior uveitis. Overall, $77.8 \%$ (7/9) of cases were treated according to the Foster protocol, and 11.1\% (1/9) each the rheumatologic and oncologic protocols. Individualized longitudinal patient data was limited but showed a $100.0 \%(4 / 4)$ rate of positive therapeutic response, with a $25.0 \%(1 / 4)$ rate of eventual disease recurrence. Information regarding tolerability of RTX was available for three patients, with one report each of $(1 / 3$, 33.3\%) gastrointestinal discomfort and fungal cellulitis attributed to RTX $[44,45]$.

\section{Scleritis (Tables 1,3 )}

There have been a total of 36 reports describing 121 patients who received treatment with RTX for noninfectious scleritis $[11,12,24,37-40,46-65,66,67-70$, $71,72,73,74]$. These patients possessed a male to female ratio of 0.56 to 1 and a mean age of $48.5 \pm 16.6$ years $\quad$ (range $=16.0$ to 81.0 years; median $=52.0$ years) when first treated with RTX. The cases of refractory non-infectious scleritis were attributed to a wide range of underlying systemic conditions including GPA (75/ $121,62.0 \%)$, RA $(15 / 121,12.4 \%)$, indeterminate etiology $(15 / 121,12.4 \%)$, antineutrophil cytoplasmic antibody (ANCA)-associated vasculitis without further specification (5/121, 4.1\%), both GPA and RA (3/121, 2.5\%), and one case each (1/121, $0.8 \%)$ of GPA and Immunoglobulin G4 (IgG4)-related disease, IgG4-related disease alone, Behçet disease, Cogan syndrome, primary Sjogren's syndrome, mixed connective tissue disease and scleroderma, microscopic polyangiitis, and HLA-B27 associated disease. Visual acuity prior to RTX therapy was reported in only 29 cases, with $62.1 \%(18 / 29)$ of patients having vision better than or equal to $20 / 40,24.1 \%(7 / 29)$ worse than or equal to $20 / 200$, and $13.8 \%$ (4/29) with vision between $20 / 40$ and 20/200.

In the 96 cases with documented treatment history, a total of 93.8\% (90/96) of patients received RTX as third-line or later therapy, followed by $4.2 \%(4 / 96)$ as second-line, and $2.1 \%(2 / 96)$ as first-line. Treatment regimens prior to RTX were either none $(2 / 94,21 . \%)$, corticosteroids alone $(4 / 94$, $4.3 \%)$, or one (30/94, 31.9\%), two (27/94, $28.7 \%)$, or three or more $(31 / 94,33.0 \%)$ corticosteroid-sparing immunosuppressive agents with or without corticosteroids. Previous immunomodulatory therapies included corticosteroids (62/90, $68.9 \%)$, cyclophosphamide (55/90, 61.1\%), methotrexate (42/ 


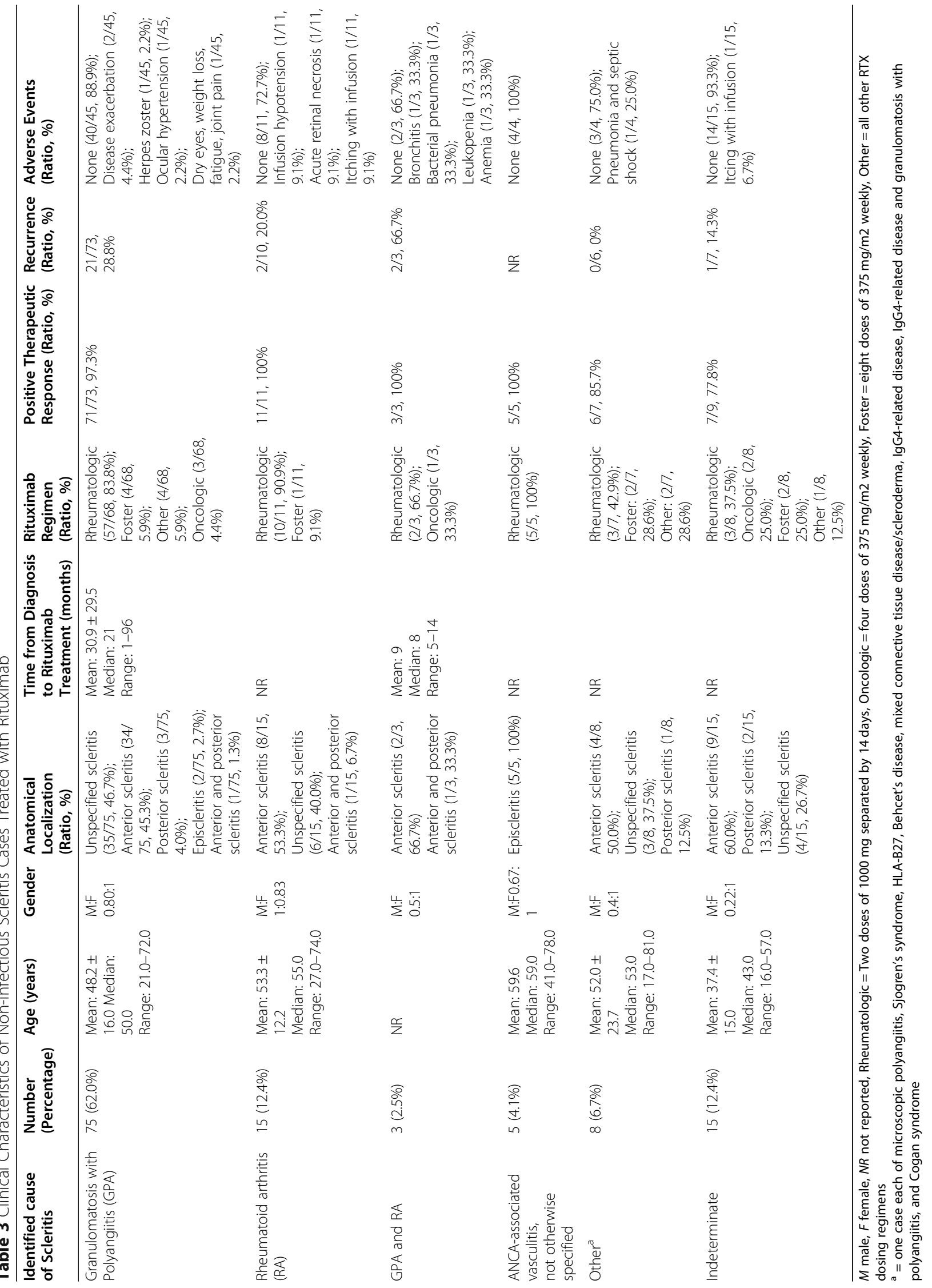


90, 46.7\%), mycophenolate mofetil (24/90, 26.7\%), azathioprine $(24 / 90,26.7 \%)$, infliximab $(12 / 90,13.3 \%)$, nonsteroidal anti-inflammatory drugs $(11 / 90,12.2 \%)$, etanercept (10/90, $11.1 \%)$, adalimumab $(9 / 90,10.0 \%)$, cyclosporine $(8 / 90,8.9 \%)$, leflunomide (6/90, 6.7\%), hydroxychloroquine (3/90, 3.3\%), sulfasalazine $(2 / 90,2.2 \%)$, intravenous immunoglobulin (2/ $90,2.2 \%)$, doxycycline $(2 / 90,2.2 \%)$, and one case each (1/90, $1.1 \%)$ of cytarabine, interferon-alpha, or golimumab, anakinra, minocycline, tocilizumab, and abatacept. The mean interval from diagnosis of scleritis to treatment with rituximab was $39.4 \pm 36.6$ months (range $=1.0$ to 168.0 months; median $=21.0$ months).

Various RTX treatment regimens were utilized to treat cases non-infectious scleritis, with 76.3\% (87/114) receiving the rheumatologic protocol, 9.6\% (11/114) receiving a variety of uncommon off-label protocols, $8.8 \%(10 / 114)$ receiving the Foster protocol, and 5.3\% (6/114) receiving the oncologic protocol. A total of $35.8 \%$ (34/95) of patients received just 1 cycle of RTX, leading to a positive response with disease remission in $84.3 \%$ (29/34), treatment failure in $14.7 \%(5 / 34)$, and disease relapse in $6.9 \%(2 / 29)$. The remaining $64.2 \%(61 / 95)$ of treated patients received between two to 15 cycles of RTX at intervals ranging from 1 to 6 months, with $18.0 \%(11 / 61)$ receiving one retreatment for disease relapse $[48,61,68,70]$. However, the rationale of designated treatment intervals and indications for retreatment in other reports were unclear. Of note, three patients developed disease recurrence despite receiving RTX treatment cycles every 4 to 6 months $[55,57]$, and two patients experienced relapse while receiving repeat infusions of unreported interval [47].

Following treatment with RTX for non-infectious scleritis, $93.3 \%(112 / 120)$ of patients experienced a positive therapeutic response, with $88.4 \%$ (99/112) experiencing disease remission for at least some period of time, and $11.6 \%(13 / 112)$ showing disease improvement based on author report. In contrast, $6.7 \%(8 / 120)$ of patients were described as having treatment failure with RTX. One or more disease recurrences eventually developed in $30.8 \%(33 / 107)$ of the patients responsive to RTX, with a mean interval to first disease recurrence of 14.4 months $(n=9$; range $=4.0$ to 55.0 months; median $=6.0$ months). In a series of 20 patients with GPA-associated scleritis treated with RTX, Joshi et al. reported a 95.0\% $(19 / 20)$ response rate followed by a $63.2 \%(12 / 19)$ rate of relapse occurring at a median of 27.0 months following treatment (mean and range unreported) [46]. In another series of 12 patients with refractory scleritis arising from rheumatoid arthritis, GPA, Cogan syndrome, or an indeterminate etiology, Suhler et al. reported a $75.0 \%(9 / 12)$ response rate following RTX treatment followed by a $77.8 \%(7 / 9)$ rate of eventual disease relapse at a mean of 32.0 weeks (range $=24.0$ to 46.0 weeks, median unreported) [48]. Information on individualized treatment regimens following uncomplicated RTX therapy was available for $83.8 \%(62 / 74)$ of patients with disease remission without recurrence, showing that $40.3 \%(25 / 62)$ continued maintenance immunosuppression with one or more non-RTX, corticosteroid-sparing systemic immunosuppressive agents at mean follow up of 18.0 months $\quad$ (range $=2.0$ to 48.0 months; median $=12.0$ months), 35.5\% (22/62) maintained ongoing RTX treatments at mean follow up of 28.9 months (range $=6.0$ to 103.0 months; median $=24.0$ months $)$, and $24.2 \%(15 / 62)$ achieved drug-free remission at mean follow up of 21.5 months $\quad$ (range $=1.0$ to 49.0 months; median $=16.0$ months). For the 26 cases that reported visual acuities following RTX treatment, $50.0 \%(20 / 40)$ of patients retained $20 / 40$ or better vision, $26.9 \%(7 / 26)$ vision between $20 / 40$ and $20 / 200$, and $23.1 \%(6 / 26)$ vision equal to or worse than $20 / 200$.

A total of $85.5 \%(71 / 83)$ reported no adverse events following treatment with RTX for non-infectious scleritis. However, two patients each $(2 / 83,2.4 \%)$ developed itching with infusion [70] and disease exacerbation [58, $62]$, and one patient each $(1 / 83,1.2 \%)$ developed herpetic mouth ulcers [37], acute retinal necrosis [67], herpes zoster [56], hypotension during infusion of RTX [55], ocular hypertension [57], pneumonia with septic shock [60], dry eyes, the combination of weight loss, fatigue, and joint pain [63], and the combination of leukopenia, anemia, bronchitis, and bacterial pneumonia [56] attributed to RTX treatments.

\section{Granulomatosis with Polyangiitis}

In total, $62.0 \%(75 / 121)$ of patients with non-infectious scleritis who received RTX treatment possessed an underlying diagnosis of GPA. These patients had a male to female ratio of 0.80 to 1 and a mean age of $48.2 \pm 16.0$ years (range $=21.0$ to 72.0 years; median $=50.0$ years $)$ when treatment began. In total, $46.7 \%$ (35/75) of these patients possessed unspecified scleritis, 45.3\% (34/75) possessed anterior scleritis, and possessed 4.0\% (3/75) posterior scleritis. The mean interval from diagnosis of scleritis to initiation of RTX treatment was 30.9 months (range $=1.0$ to 96.0 months; median $=21.0$ months). A total of $83.8 \%$ $(57 / 68)$ were treated according to the rheumatologic protocol, 5.9\% (4/68) each the Foster protocol and various uncommon off-label regimens, and 4.4\% (3/68) the oncologic protocol. A positive therapeutic response was reported for $97.3 \%$ (71/73) of treated patients, with a $28.8 \%$ (21/73) rate of subsequent disease relapse. In total, $88.9 \%$ (40/45) experienced complication free treatment, but two patients $(2 / 45,4.4 \%)$ developed disease exacerbation [58, $62]$, and one patient each $(1 / 45,2.2 \%)$ developed herpes zoster [56], ocular hypertension [57], and the nonspecific constellation of dry eyes, weight loss, fatigue, and joint pain [63] attributed to RTX treatment. 


\section{Rheumatoid arthritis}

A total of $12.4 \%(15 / 121)$ of patients received RTX as treatment for non-infectious scleritis that was attributed to RA. These patients possessed a male to female ratio of 1 to 0.83 and a mean age of 53.3 years (range $=27.0$ to 74.0 years; median $=55.0$ years) when treatment began. In total, $53.3 \%(8 / 15)$ of patients possessed anterior scleritis, $40.0 \%(6 / 15)$ possessed unspecified scleritis, and $6.7 \%(1 / 15)$ possessed both anterior and posterior scleritis. A total of $90.9 \%$ (10/11) of cases were treated according to the rheumatologic protocol and 9.1\% (1/11) by the Foster protocol. Authors reported 100\% (10/10) positive therapeutic response following RTX treatment, with a $20.0 \%(2 / 10)$ rate of eventual disease relapse. Overall, $72.7 \%(8 / 11)$ of patients experienced no adverse events following RTX treatment, but one patient each (1/11, 9.2\%) developed hypotension with infusion [55], itching with infusion [70], and acute retinal necrosis [67] attributed to RTX.

\section{Scleritis of indeterminate etiology}

In total, $12.4 \%$ (15/121) of patients with non-infectious scleritis treated with RTX possessed no discernable systemic etiology for their ocular inflammation. These patients possessed a male to female ratio of 0.22 to 1 and a mean age of 37.4 years (range $=16.0$ to 57.0 years; median $=43.0$ years $)$ when treatment began. A total of $60.0 \%(9 / 15)$ of patients were diagnosed with anterior scleritis, followed by $26.7 \%$ (4/15) with unspecified scleritis, and $13.3 \%(2 / 15)$ with posterior scleritis. Overall, $37.5 \%(3 / 8)$ of patients were treated according to the rheumatologic protocol, $25.0 \%(2 / 8)$ each according to the Foster and oncologic protocols, and 12.5\% (1/8) with an off-label regimens. Following RTX therapy, 77.8\% (7/ 9) of patients experienced a positive therapeutic response, with a $14.3 \%(1 / 7)$ rate of eventual disease recurrence. No adverse events were reported in $93.3 \%(14 / 15)$ of treated individuals, but one patient $(1 / 15,6.7 \%)$ developed itching during RTX fusion [70].

\section{Discussion}

Rituximab, a fully humanized monoclonal anti-CD20 antibody, has increasingly been utilized as a safe and efficacious treatment of refractory, non-infectious uveitis or scleritis arising from npAIR, JIA, VKH disease, Behçet disease, GPA, and RA. Rituximab was used as a third-line or later treatment in nearly three-quarters of patients with non-infectious uveitis who had been unresponsive to corticosteroid or other corticosteroid-sparing immunosuppressants, with treatment generally initiated 2 to 3 years following initial diagnosis. Half of these patients received the rheumatologic protocol, one fifth the Foster protocol, another fifth various off-label dosing regimens, and the remaining tenth the oncologic protocol. Nearly all patients with non-infectious scleritis refractory to corticosteroid or other corticosteroid sparing immunosuppressants received RTX as a third-line or later agent, with treatment generally initiated 2 to 3 years following initial diagnosis. Three quarters of these patients received the rheumatologic protocol, a tenth various uncommon off-label regimens, another tenth the Foster protocol, and the remaining with the oncologic protocol. Patients with uveitis experienced a positive therapeutic response in more than eight out of 10 cases, with less than a quarter reporting generally mild adverse events that were most commonly injection site reactions. Similarly, patients with scleritis reported disease remission in nearly nine out of 10 cases, with slightly more than a seventh of patients reporting generally mild side effects.

The efficacy of RTX for treatment of non-infectious uveitis and scleritis showed variation according to underlying systemic cause. All of the patients with noninfectious uveitis secondary to $\mathrm{VKH}$ disease $(n=12$, $100 \%)$, Behçet disease $(n=11,100 \%)$, or uveitis of indeterminate cause $(n=4,100 \%)$ had a positive therapeutic response. Similarly, nearly all patients with refractory scleritis from GPA $(n=73,97.3 \%)$, RA $(n=11,100 \%)$, GPA and RA $(n=3,100 \%)$, and unspecified ANCAassociated vasculitis $(n=5,100 \%)$ exhibited a favorable therapeutic outcome. Moderately less efficacy was observed for patients with uveitis attributed to npAIR ( $n=$ $30,80.0 \%)$, JIA $(n=21,76.2 \%)$, and sarcoidosis $(n=4$, $75.0 \%$ ), and those with scleritis from an indeterminate etiology $(n=9,77.8 \%)$. Patients with refractory uveitis from CAR showed a response in just over half $(54.5 \%)$ of patients treated with RTX, mirroring prior reports regarding the difficult nature of these conditions to control even with various modalities of long-term immunosuppression [75]. Treatment with RTX may be more efficacious for cases of non-infectious scleritis than for uveitis $(p=0.02, \mathrm{~N}-1$ two-proportion test, two-tailed). However, when patients with CAR were excluded from analysis, this difference decreased dramatically $(p=0.13, \quad \mathrm{~N}-1$ two-proportion test, two-tailed).

Maintenance of disease remission in patients with refractory non-infectious uveitis and scleritis appeared to be more likely following multiple cycles of RTX treatment or other forms of ongoing systemic immunosuppression. The majority of patients who maintained disease remission following RTX treatment either required other forms of systemic immunosuppression (uveitis: 44.4\%; scleritis: $40.3 \%$ ) or ongoing RTX infusions (uveitis: 29.3\%; scleritis: $35.5 \%$ ). A minority of patients were able to attain sustained drug free remission (uveitis: 17.8\%; scleritis: 24.2\%). Following the first cycle of RTX treatment, just under one-third of patients (uveitis: 29.6\%; scleritis: $30.8 \%$ ) developed disease recurrence.

Given that the patients in this review tended to possess either intractable non-infectious uveitis or scleritis despite treatments with corticosteroid and traditional 
non-corticosteroid immunosuppressive agents, it was unsurprising that RTX was generally used as a third-line or later medication. Other possible contributing factors could have been the cost of RTX, a relatively more difficult route of administering RTX, and/or the unfamiliarity to providers with RTX as a treatment choice in such patients. From 2005 to 2020, authors consistently used RTX as a third-line $(90 / 95,94.7 \%)$ medication to treat scleritis. While RTX was also used as predominantly a third-line medication $(67 / 90,74.4 \%)$ for treatment of refractory uveitis, some authors began to utilize RTX as a second-line $(18 / 90,20.0 \%)$ agent to treat VKH disease, npAIR, and CAR beginning in 2017 [14, 29]. Of note, RTX was used as a first line-drug for three patients with npAIR and two with CAR [14, 17, 20,35, 36]. Three of these patients were each part of a larger series wherein other patients received RTX as a third-line agent, and no explanation was provided as to why these patients differed in their treatment approach [14, 17, 20]. Sen et al. and Or et al. both initiated early treatment with RTX in hopes of targeting the B lymphocytes responsible for production of serum antibodies directed against a retinal antigen in the photoreceptor layer $[35,36]$. The generally positive outcomes noted here suggest, however, that RTX might be considered earlier in the course of therapy in some patients.

Overall, continued long-term immunosuppression appeared to be more important than selecting a particular treatment protocol given the comparable efficacy of the various treatment regimens, and the proclivity for disease relapse in RTX-treated patients with non-infectious uveitis and scleritis. The rheumatologic protocol (132/ 201, 65.7\%) was by far the most commonly utilized treatment regimen. Other established treatment regimens such as the Foster and oncologic protocols were utilized to treat a minority of patients. Roughly one in six subjects received various other off-label dosing regimens. Following RTX treatment according to the rheumatologic regimen, more than $90 \%$ of those with both uveitis and scleritis experienced a positive therapeutic response. Given limited sample sizes and lack of standardization across the studies, the collected data was insufficient to allow for comparison of treatment efficacy between the various regimens. For those cases with individualized dosing data, about one-third were treated with only 1 cycle of RTX leading to disease remission in more than eight out of 10 - although roughly one-quarter of those with initial positive therapeutic response eventually relapsed despite subsequent treatment with other forms of systemic immunomodulatory therapy. Nearly one quarter of cases reported using rituximab for 2 cycles, with half receiving a second cycle in response to disease recurrence. The remaining patients received more than 2 cycles of RTX not for specifically for disease relapse, but also for further disease control, scheduled re-treatments to maintain disease remission, or reasons not otherwise specified.

Clinical trials investigating RTX treatment for rheumatologic diseases have found it to be relatively welltolerated with mild to moderate infusion-related reactions as the most common adverse response [76-78]. Severe adverse reactions are uncommon, but can include tumor lysis syndrome, severe mucocutaneous reactions, progressive multifocal leukoencephalopathy, hepatitis B reactivation, infections, cardiac arrhythmias, renal toxicity, and bowel obstruction and perforation [79]. Roughly one-fifth $(35 / 180,19.4 \%)$ of RTX-treated patients with non-infectious uveitis and scleritis were reported to have an adverse event. According to the common terminology criteria for adverse events, $40.0 \%$ $(14 / 35)$ were Grade 1 reactions, 37.1\% (13/35) were Grade 2 reactions, and $22.8 \%(8 / 35)$ were Grade 3 or 4 reactions; no deaths (Grade 5) were reported. The most common adverse event was an infusion reaction $(11 / 35$, $31.4 \%)$. Overall, the rate of reported adverse events did not differ significantly from rates reported in the literature [76-78].

Our retrospective analysis had limitations as it relied heavily on case descriptions that varied greatly in detail regarding definition of positive therapeutic response, treatment protocols, rationale for retreatment, and total number of RTX cycles, duration of follow-up and whether disease relapse occurred, and presence of adverse events. Reports with individual case data were included, but several larger series provide only statistics rather than case descriptions and could not be incorporated into our calculations. Finally, the efficacy of the studies reviewed here may not reflect a broader population-based sample of refractory non-infectious uveitis and scleritis due to referral, selection, treatment, evaluator, and/or publication bias [80].

\section{Conclusions}

Rituximab appears to be an effective and welltolerated option for immunosuppression in patients with non-infectious uveitis and scleritis. Authors generally favored utilizing the rheumatologic protocol and tended to report mild adverse events in about one-fifth of treated patients, with infusion reactions being most common. Uveitis associated with npAIR, JIA, VKH disease, and Behçet disease, and scleritis arising from GPA and RA all appear to be well-suited for use of RTX. In contrast, roughly half of treated patients with CAR showed a positive response. Overall, the current ophthalmologic literature strongly supports RTX use for non-infectious uveitis and scleritis refractory to corticosteroid and traditional non-corticosteroid immunosuppressive agents. 


\section{Abbreviations}

ANCA: Antineutrophil cytoplasmic antibody; CAR: Cancer associated retinopathy; ERG: Electroretinography; FDA: Food and Drug Administration; GPA: Granulomatosis with polyangiitis; HLA: Human leukocyte antigen; IgG4: Immunoglobulin G4; JIA: Juvenile idiopathic arthritis; MAR: Melanoma associated retinopathy; npAIR: non-paraneoplastic autoimmune retinopathy; RTX: Rituximab; SLE: Systemic lupus erythematosus; TNF: Tumor necrosis factor; VKH: Vogt-Koyanagi-Harada

\section{Acknowledgements}

None.

\section{Authors' contributions}

CCN and AS reviewed and collected the relevant publications for review and analysis. CCN, AS, and ETC analyzed the patient data. CCN wrote the manuscript, with AS and ETC as major contributors. All authors read and approved the final manuscript.

\section{Funding}

Supported by The San Francisco Retina Foundation.

\section{Availability of data and materials}

The datasets used and/or analyzed during the current study are available from the corresponding author on reasonable request.

\section{Declarations}

Ethics approval and consent to participate

Not applicable.

\section{Consent for publication}

Not applicable.

\section{Competing interests}

The authors report no conflicts of interest. The authors alone are responsible for the content and writing of the paper.

\section{Author details}

'Department of Ophthalmology, California Pacific Medical Center, San Francisco, CA, USA. ${ }^{2}$ West Coast Retina Medical Group, 1445 Bush Street, San Francisco, CA 94109, USA. ${ }^{3}$ Department of Ophthalmology, Kaiser Permanente Santa Clara, California, USA. ${ }^{4}$ Department of Ophthalmology, Stanford University School of Medicine, Stanford, CA, USA. ${ }^{5}$ Francis I. Proctor Foundation, UCSF School of Medicine, San Francisco, CA, USA.

Received: 10 January 2021 Accepted: 18 June 2021

Published online: 16 August 2021

\section{References}

1. Cunningham ET Jr, Wender JD (2010) Practical approach to the use of corticosteroids in patients with uveitis. Can J Ophthalmol 45(4):352-358. https://doi.org/10.3129/i10-081

2. Lustig MJ, Cunningham ET Jr (2003) Use of immunosuppressive agents in uveitis. Curr Opin Ophthalmol 14(6):399-412. https://doi.org/10.1097/ 00055735-200312000-00014

3. Cunningham ET Jr, De Smet MD, Yeh S, Albini TA, Zierhut M (2015) Sustainedrelease corticosteroids for uveitis. Ocul Immunol Inflamm 14:421-424

4. Solomon SD, Cunningham ET Jr (2001) Use of corticosteroids and noncorticosteroid immunosuppressive agents in patients with uveitis. Compr Ophthalmol Updat 1(5):273-286

5. Cunningham ET, Zierhut M (2010) TNF inhibitors for uveitis: balancing efficacy and safety. Ocul Immunol Inflamm 18(6):421-423. https://doi.org/1 $0.3109 / 09273948.2010 .531176$

6. Saadoun D, Bodaghi B, Bienvenu B, Wechsler B, Sene D, Trad S, Abad S, Cacoub P, Kodjikian L, Sève P (2013) Biotherapies in inflammatory ocular disorders: interferons, immunoglobulins, monoclonal antibodies. Autoimmun Rev 12(7):774-783. https://doi.org/10.1016/j.autrev.2013.02.002

7. Maloney DG, Grillo-López AJ, White CA, Bodkin D, Schilder RJ, Neidhart JA, Janakiraman N, Foon KA, Liles TM, Dallaire BK, Wey K (1997) IDEC-C2B8 (rituximab) anti-CD20 monoclonal antibody therapy in patients with relapsed low-grade non-Hodgkin's lymphoma. Blood J Am Soc Hematol 90(6):2188-2195

8. Furst DE, Keystone EC, So AK, Braun J, Breedveld FC, Burmester GR, De Benedetti F, Dörner T, Emery P, Fleischmann R, Gibofsky A (2013) Updated consensus statement on biological agents for the treatment of rheumatic diseases, 2012. Ann Rheumatic Dis 72(suppl 2):ii2-i34

9. $\mathrm{Ng}, \mathrm{C}$, Sy A, Cunnningham, E.T., Jr., Rituximab for non-infectious orbital inflammation - in preparation

10. Standardization of Uveitis Nomenclature (SUN) Working Group (2005) Standardization of uveitis nomenclature for reporting clinical data. Results of the First International Workshop. Am J Ophthalmol 140(3) 509-516

11. Cheung CM, Murray PI, Savage CO (2005) Successful treatment of Wegener's granulomatosis associated scleritis with rituximab. Brit J Ophthalmol 89(11):1542

12. Ahmadi-Simab K, Lamprecht $P$, Nölle B, Ai M, Gross WL (2005) Successful treatment of refractory anterior scleritis in primary Sjögren's syndrome with rituximab. Ann Rheum Dis 64(7):1087-1088. https://doi.org/10.1136/ard.2 004.027128

13. Foster CS, Chang PY, Ahmed AR (2010) Combination of rituximab and intravenous immunoglobulin for recalcitrant ocular cicatricial pemphigoid: a preliminary report. Ophthalmology 117(5):861-869. https://doi.org/10.1016/j. ophtha.2009.09.049

14. Davoudi S, Ebrahimiadib N, Yasa C, Sevgi DD, Roohipoor R, Papavasilieou E, Comander J, Sobrin L (2017) Outcomes in autoimmune retinopathy patients treated with rituximab. Am J Ophthalmol 180:124-132. https://doi.org/10.1 016/j.ajo.2017.04.019

15. Fox A, Jeffrey B, Hasni S, Nussenblatt R, Sen HN (2015) Rituximab treatment for nonparaneoplastic autoimmune retinopathy. Can J Ophthalmol 50(6): e101-e104. https://doi.org/10.1016/j.jcjo.2015.08.009

16. Maleki A, Lamba N, Ma L, Lee S, Schmidt A, Foster CS (2017) Rituximab as a monotherapy or in combination therapy for the treatment of nonparaneoplastic autoimmune retinopathy. Clin Ophthalmol 11:377

17. Boudreault K, Justus S, Sengillo JD, Schuerch K, Lee W, Cabral T, Tsang $\mathrm{SH}$ (2017) Efficacy of rituximab in non-paraneoplastic autoimmune retinopathy. Orphanet J Rare Dis 12(1):129. https://doi.org/10.1186/s13 023-017-0680-7

18. Uludag G, Onal S, Arf S, Muslubas IS, Selcukbiricik F, Akbay AK, Mandel NM (2016) Electroretinographic improvement after rituximab therapy in a patient with autoimmune retinopathy. Am J Ophthalmol Case Rep 2:4-7. https://doi.org/10.1016/j.ajoc.2016.03.007

19. Eton EA, Abrams G, Khan NW, Fahim AT (2020) Autoimmune retinopathy associated with monoclonal gammopathy of undetermined significance: a case report. BMC Ophthalmol 20:1-7

20. Armbrust KR, Fox AR, Jeffrey BG, Sherry P, Sen HN. Rituximab for autoimmune retinopathy: Results of a Phase $\mathrm{I} / \mathrm{I}$ clinical trial

21. Khanna S, Martins A, Oakey Z, Mititelu M (2019) Non-paraneoplastic autoimmune retinopathy: multimodal testing characteristics of 13 cases. J Ophthalmic Inflamm Infect 9(1):6. https://doi.org/10.1186/s12348-0190171-1

22. Benson MD, Plemel DJ, Yacyshyn E, Sandhu I, MacDonald IM, Baker CF. Combination treatment with rituximab and bortezomib in a patient with non-paraneoplastic autoimmune retinopathy. Ocular Immunol Inflamm. 2020:28(6):958-65.

23. Miserocchi E, Modorati G, Berchicci L, Pontikaki I, Meroni P, Gerloni V (2016) Long-term treatment with rituximab in severe juvenile idiopathic arthritisassociated uveitis. Br J Ophthalmol 100(6):782-786. https://doi.org/10.1136/ bjophthalmol-2015-306790

24. Pelegrin L, Jakob E, Schmidt-Bacher A, Schwenger V, Becker M, Max R, Lorenz HM, Mackensen F (2014) Experiences with rituximab for the treatment of autoimmune diseases with ocular involvement. J Rheumatol 41(1):84-90. https://doi.org/10.3899/jrheum.130206

25. Heiligenhaus A, Miserocchi E, Heinz C, Gerloni V, Kotaniemi K (2011) Treatment of severe uveitis associated with juvenile idiopathic arthritis with anti-CD20 monoclonal antibody (rituximab). Rheumatology 50(8):1390-1394. https://doi.org/10.1093/rheumatology/ker107

26. Calvo-Río V, Santos-Gómez M, Calvo I, González-Fernández MI, LópezMontesinos B, Mesquida M, Adán A, Hernández MV, Maíz O, Atanes A, Bravo B (2017) Anti-interleukin-6 receptor tocilizumab for severe juvenile idiopathic arthritis-associated uveitis refractory to anti-tumor necrosis factor therapy: a multicenter study of twenty-five patients. Arthritis Rheumatol 69(3):668-675. https://doi.org/10.1002/art.39940 
27. Umran RM, Shukur ZY (2018) Rituximab for sight-threatening refractory pediatric Vogt-Koyanagi-Harada disease. Mod Rheumatol 28(1):197-199. https://doi.org/10.3109/14397595.2015.1071234

28. Dolz-Marco R, Gallego-Pinazo R, Díaz-Llopis M (2011) Rituximab in refractory Vogt-Koyanagi-Harada disease. J Ophthalmic Inflamm Infect 1(4):177-180. https://doi.org/10.1007/s12348-011-0027-9

29. Abu El-Asrar AM, Dheyab A, Khatib D, Struyf S, Van Damme J, Opdenakker G (2020) Efficacy of B cell depletion therapy with rituximab in refractory chronic recurrent uveitis associated with Vogt-Koyanagi-Harada disease. Ocul Immunol Inflamm 27:1-8

30. Caso F, Fiocco U, Costa L, Sfriso P, Punzi L, Doria A (2014 Mar) Successful use of rituximab in a young patient with immunoglobulin G4-related disease and refractory scleritis. Joint Bone Spine 81(2):190-192. https://doi. org/10.1016/j.jbspin.2013.07.011

31. Davatchi F, Shams H, Rezaipoor M, SADEGHI-ABDOLLAHI B, Shahram F, Nadji A, CHAMS-DAVATCHI C, Akhlaghi M, Faezi T, Naderi N (2010) Rituximab in intractable ocular lesions of Behcet's disease; randomized single-blind control study (pilot study). Int J Rheum Dis 13(3):246-252. https://doi.org/10.1111/j.1756-185X.2010.01546.x

32. Sadreddini S, Noshad H, Molaeefard M, Noshad R (2008) Treatment of retinal vasculitis in Behcet's disease with rituximab. Mod Rheumatol 18(3): 306-308. https://doi.org/10.3109/s10165-008-0057-9

33. Dy I, Chintapatla R, Preeshagul I, Becker D (2013) Treatment of cancerassociated retinopathy with rituximab. J Natl Compr Cancer Netw 11(11): 1320-1324. https://doi.org/10.6004/jnccn.2013.0156

34. Mahdi N, Faia LJ, Goodwin J, Nussenblatt RB, Nida SH (2010) A case of autoimmune retinopathy associated with thyroid carcinoma. Ocul Immunol Inflamm 18(4):322-323. https://doi.org/10.3109/09273941003802379

35. Sen HN, Chan CC, Caruso RC, Fariss RN, Nussenblatt RB, Buggage RR (2004) Waldenström's macroglobulinemia-associated retinopathy. Ophthalmology 111(3):535-539. https://doi.org/10.1016/j.ophtha.2003.05.036

36. Or C, Collins DR, Merkur AB, Wang Y, Chan CC, Forooghian F (2013) Intravenous rituximab for the treatment of cancer-associated retinopathy. Can J Ophthalmol 48(2):e35-e38. https://doi.org/10.1016/j. jcjo.2012.11.010

37. Lower EE, Baughman RP, Kaufman AH (2012) Rituximab for refractory granulomatous eye disease. Clin Ophthalmol 6:1613

38. Ahmed A, Foster CS (2019) Cyclophosphamide or rituximab treatment of scleritis and uveitis for patients with granulomatosis with polyangiitis. Ophthalmic Res 61(1):44-50. https://doi.org/10.1159/000486791

39. Sadhu S, Majumder PD, Biswas J (2020) Biological therapy in refractory cases of uveitis and scleritis: an analysis of 18 cases from a tertiary eye care center from South India. Indian J Ophthalmol 68(9):1929-1933. https://doi.org/1 0.4103/ijo.IJO_966_20

40. Lasave AF, You C, Ma L, Abusamra K, Lamba N, Navarro MV, Meese H, Foster CS (2018) Long-term outcomes of rituximab therapy in patients with noninfectious posterior uveitis refractory to conventional immunosuppressive therapy. Retina 38(2):395-402. https://doi.org/10.1097/ IAE.0000000000001563

41. Tomkins-Netzer O, Taylor SR, Lightman S (2013) Can rituximab induce longterm disease remission in patients with intra-ocular non-infectious inflammation? Ophthalmologica 230(3):109-115. https://doi.org/10.1159/ 000351426

42. Babu K, Murthy GJ, Murthy KR (2020) Uveitis, retinal vasculitis, and loss of accommodation as sole manifestations of demyelinating disease: a case report. Indian J Ophthalmol 68(9):2007-2009. https://doi.org/10.4103/ijo. IJO_2017_19

43. Nicholson L, Sobrin L (2013) Anterior uveitis secondary to type II essential cryoglobulinemia. J Ophthalmic Inflamm Infect 3(1):56. https://doi.org/10.11 86/1869-5760-3-56

44. Cornish KS, Kuffova L, Forrester JV (2015) Treatment of diffuse subretinal fibrosis uveitis with rituximab. Br J Ophthalmol 99(2):153-154. https://doi. org/10.1136/bjophthalmol-2013-304686

45. Tappeiner C, Heinz C, Specker C, Heiligenhaus A (2007) Rituximab as a treatment option for refractory endogenous anterior uveitis. Ophthalmic Res 39(3):184-186. https://doi.org/10.1159/000103239

46. Joshi L, Tanna A, McAdoo SP, Medjeral-Thomas N, Taylor SR, Sandhu G, Tarzi RM, Pusey CD, Lightman S (2015) Long-term outcomes of rituximab therapy in ocular granulomatosis with polyangiitis: impact on localized and nonlocalized disease. Ophthalmology 122(6):1262-1268. https://doi.org/10.1 016/j.ophtha.2015.01.016
47. Recillas-Gispert C, Serna-Ojeda JC, Flores-Suárez LF (2015) Rituximab in the treatment of refractory scleritis in patients with granulomatosis with polyangiitis (Wegener's). Graefes Arch Clin Exp Ophthalmol 253(12):22792284. https://doi.org/10.1007/s00417-015-3198-5

48. Suhler EB, Lim LL, Beardsley RM, Giles TR, Pasadhika S, Lee ST, de Saint Sardos A, Butler NJ, Smith JR, Rosenbaum JT (2014) Rituximab therapy for refractory scleritis: results of a phase $1 / / 1$ dose-ranging, randomized, clinical trial. Ophthalmology 121(10):1885-1891. https://doi.org/10.1016/j.ophtha.2014.04.044

49. Dalkilic E, Alkis N, Kamali S (2012) Rituximab as a new therapeutic option in granulomatosis with polyangiitis: a report of two cases. Mod Rheumatol 22(3):463-466. https://doi.org/10.3109/s10165-011-0507-7

50. Morarji J, Joshi L, Tomkins-Netzer O, Lightman S, Taylor SR (2012) Combined infliximab and rituximab in necrotising scleritis. Case Rep Ophthalmol 3(3): 286-290. https://doi.org/10.1159/000342842

51. Huerva V, Sanchez MC, Traveset A, Jurjo C, Ruiz A (2010) Rituximab for peripheral ulcerative keratitis with Wegener granulomatosis. Cornea 29(6): 708-710. https://doi.org/10.1097//CO.0b013e3181c296ed

52. Taylor SR, Salama AD, Joshi L, Pusey CD, Lightman SL (2009) Rituximab is effective in the treatment of refractory ophthalmic Wegener's granulomatosis. Arthritis Rheumatism 60(5):1540-1547. https://doi.org/10.1002/art.24454

53. Onal S, Kazokoglu $H$, Koç A, Yavuz S (2008) Rituximab for remission induction in a patient with relapsing necrotizing scleritis associated with limited Wegener's granulomatosis. Ocul Immunol Inflamm 16(5-6):230-232. https://doi.org/10.1080/09273940802331643

54. Freidlin J, Wong IG, Acharya N (2007) Rituximab treatment for peripheral ulcerative keratitis associated with Wegener's granulomatosis. Brit J Ophthalmol 91(10):1414

55. Cao JH, Oray M, Cocho L, Foster CS (2016) Rituximab in the treatment of refractory noninfectious scleritis. Am J Ophthalmol 164:22-28. https://doi. org/10.1016/j.ajo.2015.12.032

56. You C, Ma L, Lasave AF, Foster CS (2018) Rituximab induction and maintenance treatment in patients with scleritis and granulomatosis with polyangiitis (Wegener's). Ocul Immunol Inflamm 26(8):1166-1173. https:// doi.org/10.1080/09273948.2017.1327602

57. Mohanty G, Dash S (2020) Rituximab induced increased intraocular pressure in recalcitrant Scleritis. J Case Rep 10(1):8-11

58. Babu K, Dharmanand BG (2020) Worsening of posterior scleritis and orbital pseudotumor in a patient with granulomatosis polyangiitis with rituximab-a case report. Indian J Ophthalmol 68(9):1986-1988. https://doi.org/10.4103/ ijo.IJO_1644_20

59. Fujita Y, Fukui S, Endo Y, Tsuji S, Takatani A, Shimizu T, Umeda M, Nishino A, Koga T, Kawashiri SY, Iwamoto N, Ichinose K, Tamai M, Nakamura H, Origuchi T, Ueki R, Uematsu M, Ishida K, Abe K, Kawakami A (2018) Peripheral Ulcerative Keratitis Associated with Granulomatosis with Polyangiitis Emerging Despite Cyclophosphamide, Successfully Treated with Rituximab. Intern Med. 57(12): 1783-1788. https://doi.org/10.2169/internalmedicine.0215-17

60. Fabiani C, Sota J, Sainz-de-la-Maza M, Pelegrín L, Emmi G, Lopalco G, lannone F, Vannozzi L, Guerriero S, Gelmi MC, Rigante D (2020) New potential weapons for refractory scleritis in the era of targeted therapy. Mediat Inflamm 17:2020

61. Shenavandeh S, Petramfar P (2017) Three atypical manifestations of granulomatosis with polyangiitis: lateral medullary syndrome, anterior cheek mass and melting scleritis of eye. Reumatologia 55(3):145-150. https://doi. org/10.5114/reum.2017.68915

62. Shih CB, Wang YC, Lai CC (2019) Ocular and orbital exacerbation after rituximab therapy for granulomatosis with polyangiitis. Can J Ophthalmol 54(5):e237-e241. https://doi.org/10.1016/j.jcjo.2018.12.004

63. Rivera-Grana E, Siak J, Rosenbaum JT (2018) Use of biologic response modifiers for the management of ocular inflammatory conditions. Indian J Rheumatol 13(4):259

64. Asín MA, Charles P, Rothschild PR, Terrier B, Brézin A, Mouthon L, Guillevin L, Puéchal X (2019) Ocular involvement in granulomatosis with polyangiitis: a single-center cohort study on 63 patients. Autoimmun Rev 18(5):493-500. https://doi.org/10.1016/j.autrev.2019.03.001

65. Alfawaz AM (2016) Successful cataract surgery in a patient with refractory Wegener's granulomatosis effectively treated with rituximab: a case report. Saudi J Ophthalmol 30(3):194-197. https://doi.org/10.1016/j.sjopt.2016.02.002

66. Murthy SI, Shah S, Bagga B, Dudam R (2020) Rituximab therapy combined with methotrexate for severe necrotizing scleritis in a case of granulomatosis with polyangiitis. Indian J Ophthalmol 68(9):1981-1983. https://doi.org/10.4103/ijo.IJO_2249_19 
67. Schuler S, Brunner M, Bernauer W (2016) Rituximab and acute retinal necrosis in a patient with scleromalacia and rheumatoid arthritis. Ocul Immunol Inflamm 24(1): 96-98. https:/doi.org/10.3109/09273948.2014.999377

68. laccheri B, Androudi S, Bocci EB, Gerli R, Cagini C, Fiore T (2010) Rituximab treatment for persistent scleritis associated with rheumatoid arthritis. Ocul Immunol Inflamm 18(3):223-225. https://doi.org/10.3109/09273941003739928

69. Chauhan S, Kamal A, Thompson RN, Estrach C, Moots RJ (2009) Rituximab for treatment of scleritis associated with rheumatoid arthritis. $\mathrm{Br} J$ Ophthalmol 93(7):984-985. https://doi.org/10.1136/bjo.2008.147157

70. Kurz PA, Suhler EB, Choi D, Rosenbaum JT (2009) Rituximab for treatment of ocular inflammatory disease: a series of four cases. Br J Ophthalmol 93(4): 546-548. https://doi.org/10.1136/bjo.2007.133173

71. Della-Torre E, Lanzillotta M, Campochiaro C, Bozzalla E, Bozzolo E, Bandiera A, Bazzigaluppi E, Canevari C, Modorati G, Stone JH, Manfredi A, Doglioni C (2016) Antineutrophil cytoplasmic antibody positivity in lgG4-related disease: A case report and review of the literature. Medicine (Baltimore) 95(34):e4633. https://doi.org/10.1097/MD.0000000000004633

72. Bogdanic-Werner K, Fernandez-Sanz G, Alejandre Alba N, Ferrer Soldevila P, Romero-Bueno Fl, Sanchez-Pernaute O (2013) Rituximab therapy for refractory idiopathic scleritis. Ocul Immunol Inflamm 21(4):329-332. https:// doi.org/10.3109/09273948.2013.788724

73. Kasi SK, Kim HJ, Basham RP, Cunningham ET Jr, Sy A, Lustig L, Kersten RC (2016) Idiopathic orbital inflammation associated with necrotizing scleritis and temporal bone inflammation. Ophthalmic Plast Reconstr Surg 32(4): e77-e79. https://doi.org/10.1097//OP.0000000000000251

74. Caso F, Rigante D, Vitale A, Costa L, Bascherini V, Latronico E, Franceschini R Cantarini L (2015) Long-lasting uveitis remission and hearing loss recovery after rituximab in Vogt-Koyanagi-Harada disease. Clin Rheumatol 34(10): 1817-1820. https://doi.org/10.1007/s10067-014-2781-1

75. Grewal DS, Fishman GA, Jampol LM (2014) Autoimmune retinopathy and antiretinal antibodies: a review. Retina 34(5):1023-1041. https://doi.org/10.1 097/01.iae.0000450880.26367.4e

76. van Vollenhoven RF, Emery P, Bingham CO, Keystone EC, Fleischmann R, Furst DE, Macey K, Sweetser M, Kelman A, Rao R (2010) Longterm safety of patients receiving rituximab in rheumatoid arthritis clinical trials. J Rheumatol 37(3):558-567. https://doi.org/10.3899/jhrheum.090856

77. Van Vollenhoven RF, Emery P, Bingham CO, Keystone EC, Fleischmann RM, Furst DE, Tyson N, Collinson N, Lehane PB (2013) Long-term safety of rituximab in rheumatoid arthritis: 9.5-year follow-up of the global clinical trial programme with a focus on adverse events of interest in RA patients. Ann Rheum Dis 72(9):1496-1502. https://doi.org/10.1136/annrheumdis-2 012-201956

78. Cohen SB, Emery P, Greenwald MW, Dougados M, Furie RA, Genovese MC Keystone EC, Loveless JE, Burmester GR, Cravets MW, Hessey EW (2006) Rituximab for rheumatoid arthritis refractory to anti-tumor necrosis factor therapy: results of a multicenter, randomized, double-blind, placebocontrolled, phase III trial evaluating primary efficacy and safety at twentyfour weeks. Arthritis Rheumatism 54(9):2793-2806. https://doi.org/10.1002/a rt.22025

79. Genentech Inc (2010) Rituxan (rituximab) [package insert]. Genentech Inc, South San Francisco

80. Cunningham Jr ET, Acharya N, Kempen JH, Zierhut M. Design and interpretation of clinic-based studies in uveitis

\section{Publisher's Note}

Springer Nature remains neutral with regard to jurisdictional claims in published maps and institutional affiliations.

\section{Submit your manuscript to a SpringerOpen ${ }^{\circ}$ journal and benefit from:}

- Convenient online submission

- Rigorous peer review

- Open access: articles freely available online

- High visibility within the field

- Retaining the copyright to your article

Submit your next manuscript at $\boldsymbol{\nabla}$ springeropen.com 